\title{
Neonatal Thyrotoxicosis with Tricuspid Valve Regurgitation and Hydrops in a Preterm Infant Born to a Mother with Graves' Disease
}

\author{
Stefani Doucette, MD ${ }^{1}$ Anne Tierney, FRCPC ${ }^{2}$ Anne Roggensack, FRCSC ${ }^{3}$ Kamran Yusuf, FAAP ${ }^{2}$ \\ ${ }^{1}$ Department of Pediatrics, Children's Hospital of Eastern Ontario, \\ Ottawa, Canada \\ 2 Section of Neonatology, Department of Pediatrics, University of \\ Calgary, Cumming School of Medicine, Alberta, Canada \\ ${ }^{3}$ Department of Obstetrics and Gynecology, University of Calgary,

\begin{abstract}
Address for correspondence Kamran Yusuf, FAAP, Section of Neonatology, Department of Pediatrics, University of Calgary, Cumming School of Medicine, Room 273, Heritage Medical Research Building, 3330 Hospital Drive NW, Calgary, Alberta T2N 4N1, Canada (e-mail: kyusuf@ucalgary.ca).
\end{abstract} Cumming School of Medicine, Alberta, Canada

Am J Perinatol Rep 2018;8:e85-e88.

\begin{abstract}
Keywords

- thyrotoxicosis

- fetal

- neonatal

- tricuspid regurgitation

- hydrops

Neonatal hyperthyroidism is rare disorder due to the passage of thyroid receptor antibodies (TRBs) from the mother to the fetus. Neonatal thyrotoxicosis can present in several ways and if unrecognized, can be fatal. We present a preterm neonate who developed fetal hydrops and tricuspid regurgitation in utero. The mother had a history of treated Grave's disease. The infant responded to maternal treatment antenatally and postnatal anti-thyroid treatment, with resolution of both the tricuspid regurgitation and hydrops. To our knowledge, this is the first case report of tricuspid regurgitation associated with fetal and neonatal thyrotoxicosis. Our case also highlights the importance of obtaining a detailed and accurate history in a mother with previous Grave's disease, even if treated.
\end{abstract}

Neonatal hyperthyroidism is an uncommon but potentially fatal disorder with an incidence of 1 in 50,000 infants. The disease occurs almost exclusively secondary to the transplacental passage of thyroid receptor antibodies (TRABs) to the fetus from a mother with Graves' disease or rarely Hashimoto's thyroiditis. ${ }^{1}$ These antibodies bind to the thyroid-stimulating hormone (TSH) receptors on the fetal thyroid, stimulating the gland to produce excessive thyroxine resulting in thyrotoxicosis. The incidence of thyrotoxicosis during pregnancy is 0.1 to $-0.4 \%$ with 1 to $5 \%$ of infants of these mothers developing thyrotoxicosis, the manifestations of which can begin in utero. ${ }^{2}$ Fetal thyrotoxicosis presents in several ways including tachycardia, intrauterine growth retardation, goiter, cardiac failure, intrauterine death, and non-immune hydrops. ${ }^{1,3}$ We describe a neonate with thyrotoxicosis, who developed nonimmune hydrops and severe tricuspid regurgitation in utero, both of which responded to maternal treatment initially and later neonatal treatment. To the best of our knowledge, this is

received

October 18, 2017

accepted after revision

January 15, 2018 10.1055/s-0038-1645879. ISSN 2157-6998. the first report of tricuspid regurgitation associated with fetal and neonatal thyrotoxicosis.

\section{Case Report}

A 36-year-old, gravida 2 para 1, mother presented to hospital in a thyrotoxic state with exophthalmos. She had a history of Graves' disease diagnosed 15 years before during the postpartum period of her first pregnancy. At that time, she was treated with carbimazole and subsequently had a subtotal thyroidectomy in 2001, performed in her home country. The patient immigrated to Canada in 2013. A thyroid ultrasound done in 2014 showed a significant amount of thyroid tissue remaining. During this pregnancy, she did not seek medical care until late in the second trimester at 26 weeks' gestation. At presentation, the mother complained of decreased fetal movements, and the fetus was noted to be persistently tachycardic. As medical reports from her home country were not available,
Copyright $\odot 2018$ by Thieme Medical Publishers, Inc., 333 Seventh Avenue, New York, NY 10001, USA. Tel: +1(212) 584-4662.
License terms

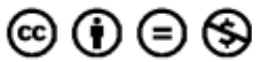


her previous history of hyperthyroidism was obtained after a detailed and meticulous history. She had negative serology for hepatitis B, human immunodeficiency virus (HIV), and syphilis and was immune to rubella and varicella. There was no smoking, drug use, or alcohol use during the pregnancy. Both her T3 and T4 levels were elevated, and TRAB was reported as $472 \mathrm{IU} / \mathrm{L}$ (normal < 1.75). TSH was $<0.01 \mathrm{mIU} / \mathrm{L}$ (normal 0.3-6.20).

On antenatal ultrasound, the fetus was hydropic with bilateral pleural effusions, ascites, a pericardial effusion, and body wall edema (-Fig. 1). On fetal echocardiography, the fetus was noted to have tricuspid regurgitation with tricuspid valve dysplasia and right atrial enlargement. Persistent fetal tachycardia of 170 to 185 beats/min was also present. A fetal magnetic resonance imaging (MRI) was significant for mild cerebral ventriculomegaly, fetal hydrops, and a fetal goiter. Although fetal thyrotoxicosis was the primary diagnosis, the presence of abnormal central nervous system (cerebral ventriculomegaly) and cardiovascular (tricuspid valve dysplasia and regurgitation) findings raised the possibility of a syndrome or chromosomal disorder. Amniocentesis, however, revealed a normal female karyotype. Screening for (T)oxoplasmosis, (O)ther Agents, (R)ubella (also known as German Measles), (C)ytomegalovirus, and (H)erpes Simplex (TORCH) infections was negative.

The mother was admitted to hospital and was started on anti-thyroid medications, methimazole, propranolol, and Syn- throid, employing a "block and replace" strategy. Initially, because of the poor prognosis of fetal hydrops, the uncertainty of the diagnosis, and the possibility of development of maternal "mirror syndrome," discussions were held with the family on possible options for management. The parents wanted to continue with the pregnancy and fully support the fetus. The patient received antenatal corticosteroids at 27 weeks of gestation for threatened preterm labor. Fetal tachycardia resolved within 24 hours following propranolol treatment. Daily fetal ultrasound evaluations were performed. After 8 days of maternal anti-thyroid treatment, fetal hydrops showed improvement, but the thyroid enlargement and tricuspid regurgitation persisted unchanged ( - Fig. 2). Given that that the mother's condition had improved, and the fetus was stable, the patient was allowed to go home on a pass.

At an estimated gestational age of 30 weeks and 4 days, the mother presented to a community hospital in preterm labor. Labor was advanced, and transfer to a center with level III neonatal intensive care facility was considered unsafe. Seven hours after the onset of labor, a baby girl was delivered vaginally. APGAR scores were 5 and 7 at 1 and 5 minutes, respectively, and the birthweight was $2030 \mathrm{~g}$. Because of poor respiratory effort and increasing oxygen requirements, she was intubated and placed on a mechanical ventilator. Following stabilization, she was transferred to the regional Level III neonatal intensive care unit.

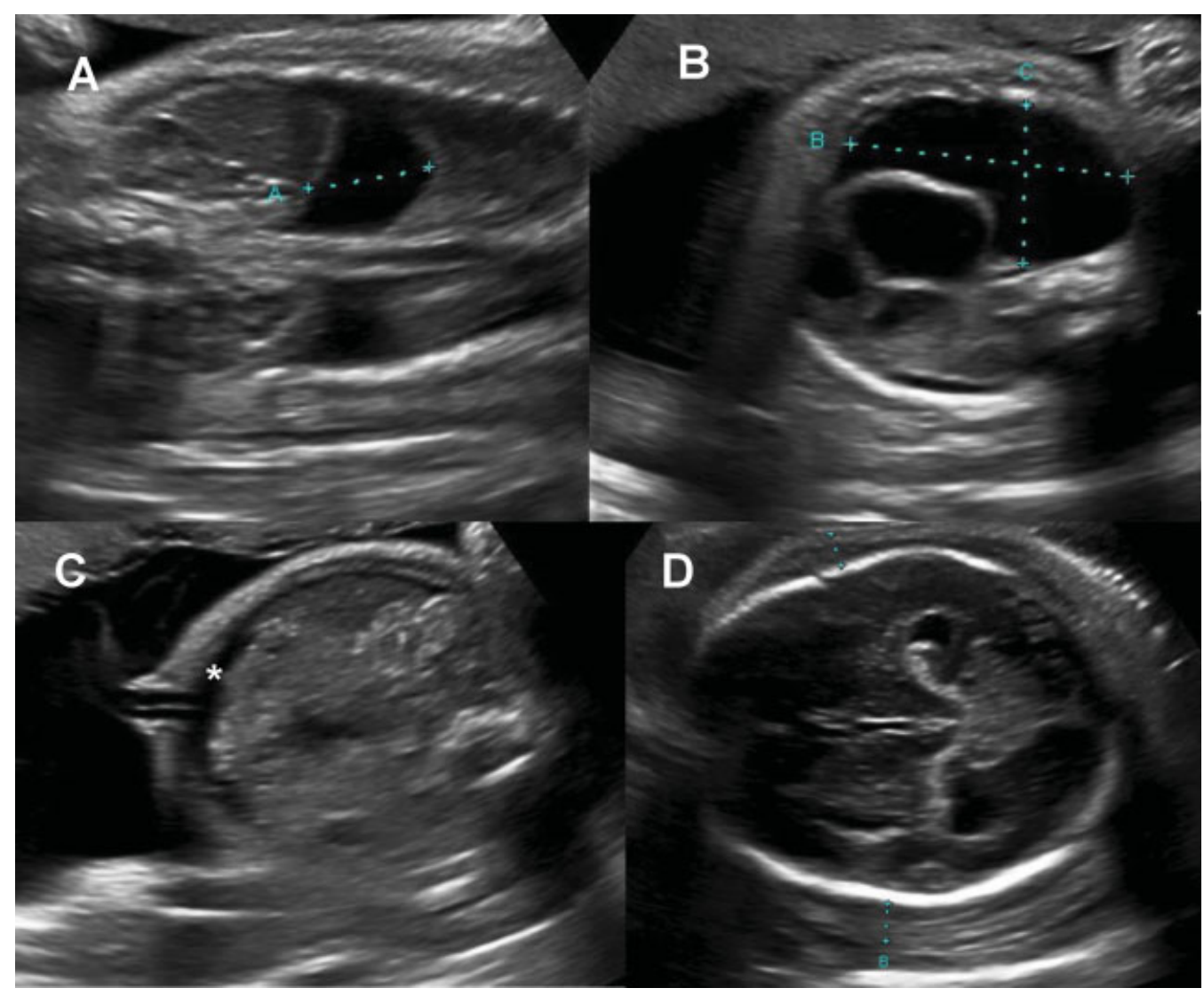

Fig. 1 Fetal ultrasound at 27 weeks gestational age demonstrating hydrops fetalis. (A) Longitudinal fetal view demonstrating bilateral pleural effusions. (B) Transverse view of fetal chest demonstrating large right pleural effusion. (C) Transverse view of fetal abdomen demonstrating ascites (asterisk). (D) Transverse view of fetal head demonstrating skin edema of 7 to $8 \mathrm{~mm}$. 


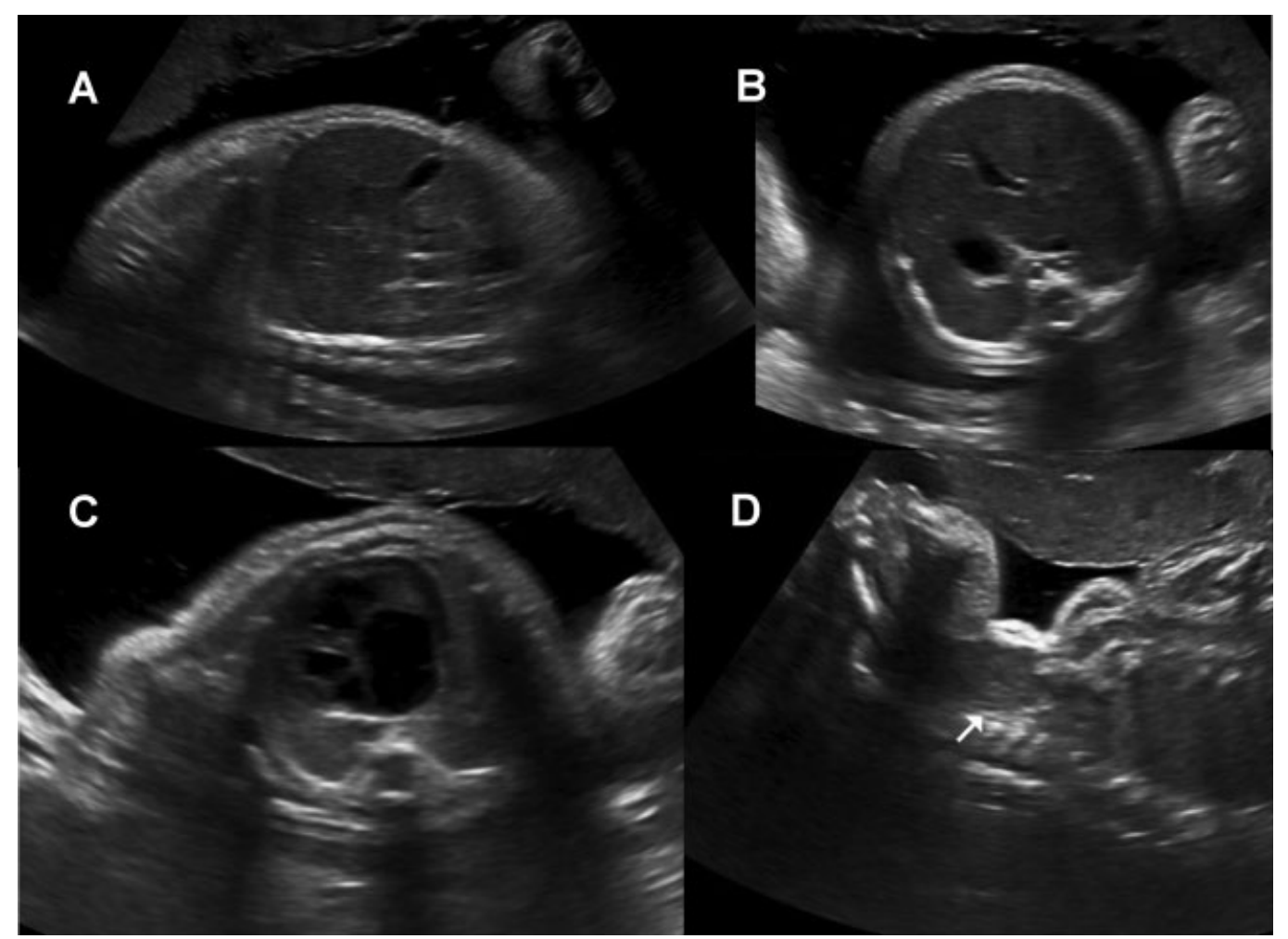

Fig. 2 Fetal ultrasound at 30 weeks gestational age with resolution of hydrops fetalis and presence of thyromegaly. (A) Longitudinal fetal view demonstrating resolution of pleural effusions. (B) Transverse view of fetal abdomen demonstrating resolution of ascites. (C) Transverse view of fetal chest demonstrating resolution of pleural effusions. (D) Longitudinal view of the fetal neck demonstrating extension of the fetal neck and enlarged fetal thyroid (arrow).

Physical examination of the infant at birth was significant for exophthalmos and a palpable thyroid gland, but no obvious evidence of goiter. There was no evidence of hydrops, and the chest X-ray did not show any pleural effusion. Postnatal echocardiography showed moderate to severe tricuspid valve regurgitation, right ventricular, and atrial dilation with no evidence of pericardial effusion. There was evidence of pulmonary hypertension with the pulmonary pressures the same as the systemic arterial pressure. However, other than mild tachycardia, she had no signs of cardiac failure. She did not require any cardiac support or treatment for the pulmonary hypertension. She was extubated at 8 hours of life and remained stable on room air. Postnatal head ultrasound showed mild bilateral lateral ventriculomegaly and no other intracranial anomalies. Thyroid ultrasound showed mild thyroid enlargement with thyroid size $1.1 \times 1.2 \times 1.9 \mathrm{~cm}$ for the right and $1.4 \times 0.8 \times 2.5 \mathrm{~cm}$ for the left thyroid lobe. There was also marked hyperemia of the thyroid gland.

Initial bloodwork on day 1 of life revealed a TSH $<0.01 \mathrm{mIU} / \mathrm{L}$ (normal 11.00-36.00 mIU/L). Free T4 was $20.2 \mathrm{pmol} / \mathrm{L}$ (normal range $10.0-25.0 \mathrm{pmol} / \mathrm{L}$ ). On the second day of life, free T4 was $>100 \mathrm{pmol} / \mathrm{L}$ and outside the measurable reference of the laboratory assay. TRAB levels were $208.4 \mathrm{IU} / \mathrm{L}$. The infant was started on methimazole at a dose of $0.3 \mathrm{mg}$ TID. Levels of free T4 gradually came down, and the dose of methimazole was gradually reduced. The exophthalmos and tachycardia improved, the infant fed well, gained weight, and was discharged on methimazole $0.3 \mathrm{mg}$ twice a day.
At 8 weeks of life, an echocardiogram showed complete resolution of the tricuspid regurgitation and normal right atrial and ventricle size with good biventricular function. The mild cerebral ventriculomegaly was stable, and the infant continues to gain weight with normal head circumference.

\section{Discussion}

The management of this case was challenging for several reasons. The mother had her initial medical and surgical treatment for hyperthyroidism prior to her arrival in Canada, and her detailed medical information was not available. In addition, she had no antenatal care until 26 weeks of gestation, and initial antenatal ultrasound findings were difficult to interpret. The presence of cardiac and central nervous system abnormalities raised the possibility of an underlying genetic condition in addition to the fetal thyrotoxicosis. Importantly, tricuspid regurgitation is reported with fetal thyrotoxicosis.

Our case report highlights several important features related to fetal and neonatal thyrotoxicosis. Although tricuspid regurgitation has been described in adults with thyrotoxicosis, we believe this is the first report in fetal and neonatal thyrotoxicosis. ${ }^{4}$ The cause of tricuspid regurgitation in hyperthyroidism is not fully understood. It is postulated that the tachycardia and high output state in hyperthyroidism results in greater volume load and more rapid venous return to the right atrium and ventricle. The increased volume can elevate pulmonary arterial pressure and result in right ventricle 
dilation. Tricuspid regurgitation can occur due to dilation of the tricuspid annulus. Whether the mechanism for tricuspid regurgitation in adults also plays a role in the fetus and newborn is, however, not established. ${ }^{5,6}$ As described in adults, our patient had right ventricular dilation with pulmonary hypertension and responded to anti-thyroid treatment with complete resolution of tricuspid regurgitation and right ventricle dilation.

Non-immune hydrops fetalis is reported with fetal thyrotoxicosis. To our knowledge, this is the third case report where hydrops completely resolved after maternal treatment of hyperthyroidism. ${ }^{7,8}$ Although non-immune hydrops is generally a condition with a grave prognosis, our and other case reports suggest that the prognosis of hydrops may not be as bad in fetal thyrotoxicosis as in other causes of nonimmune hydrops. ${ }^{9}$ This highlights the importance of early diagnosis so that maternal treatment can be instituted as the transfer of TRABs to the fetus via the placenta increases with increasing gestation with maximum transfer taking place in the third trimester. ${ }^{3,10}$ Other features of fetal thyrotoxicosis such as tachycardia and goiter have also been reported to respond to maternal treatment. ${ }^{11}$ In our case, a "block and replace" strategy was employed in which the mother was started on propranolol, methimazole, and Synthroid. Antithyroid drugs, such as methimazole and propranolol, readily cross the placenta but there is very little if any passage of thyroid hormones. As most symptoms of fetal thyrotoxicosis, including tachycardia, are mediated by the increased adrenergic response, $\beta$-adrenergic blocking medications such as propranolol can ameliorate these symptoms within a few hours of their administration. In contrast, due to the long half-life of circulating T4 in neonates (3-4 days), little or no response to anti-thyroid medications alone may be seen in the first few days of initiating anti-thyroid therapy.

Although secondary to maternal Graves' disease, fetal thyrotoxicosis is independent of maternal disease state. The mother may be hyperthyroid, euthyroid, and even hypothyroid after earlier treatment for Graves' disease. This underscores the importance of obtaining a proper and detailed history from the mother and thyroid function screening along with TRAB levels in a pregnant women with a previous history of Graves' disease even if treated. ${ }^{2}$ Higher TRAB levels in the mother are associated with a greater risk of thyrotoxicosis in the fetus. The mother in our case developed hyperthyroidism despite a sub-total thyroidectomy. In such cases, the mother's thyroid hormones should be monitored every 2 to 6 weeks with aim of keeping the free $\mathrm{T} 4$ at or just above the reference normal range. The fetus can be monitored by heart rate, growth, amniotic fluid volume, and the development of goiter by means of serial ultrasounds. Monitoring of fetal thyroid hormone and TRAB levels by cord blood sampling has also been recommended, but carries a small but definite risk of preterm labor. ${ }^{12}$
Following birth, symptoms of thyrotoxicosis may be delayed by a few days as the anti-thyroid medications are cleared from the infant's circulation. The natural history of neonatal hyperthyroidism secondary to maternal Graves' disease is gradual spontaneous resolution over time as maternal TRABs levels slowly decrease in the infant's circulation. Goiter if present at the time of delivery will resolve slowly over the course of several months. These infants need close follow-up by pediatricians and endocrinologists with regular monitoring of their thyroid function and TRAB levels.

In summary, fetal and neonatal thyrotoxicosis carries a high risk of mortality and significant morbidity. However, with early diagnosis and treatment, the outlook for these infants can be vastly improved. The importance of early and regular antenatal care and a proper and detailed history in pregnant women cannot be over emphasized.

Conflict of Interest

The authors have no conflict of interest, financial or otherwise.

\section{References}

1 Ogilvy-Stuart AL. Neonatal thyrotoxicosis. Neoreviews 2017;18 (07):e422-e427

2 Correia MF, Maria AT, Prado S, Limbert C. Neonatal thyrotoxicosis caused by maternal autoimmune hyperthyroidism. BMJ Case Rep 2015;2015:bcr201409283

3 Chan GW, Mandel SJ. Therapy insight: management of Graves' disease during pregnancy. Nat Clin Pract Endocrinol Metab 2007; 3(06):470-478

4 Park JH, Shong M, Lee JH, Choi SW, Jeong JO, Seong IW. Reversible severe tricuspid regurgitation with right heart failure associated with thyrotoxicosis. Thyroid 2006;16(08):813-814

5 Xenopoulos NP, Braden GA, Applegate RJ. Severe right heart failure in a patient with Grave's disease. Clin Cardiol 1996;19(11):903-905

6 Nakchbandi IA, Wirth JA, Inzucchi SE. Pulmonary hypertension caused by Graves' thyrotoxicosis: normal pulmonary hemodynamics restored by (131)I treatment. Chest 1999;116(05):1483-1485

7 Treadwell MC, Sherer DM, Sacks AJ, Ghezzi F, Romero R. Successful treatment of recurrent non-immune hydrops secondary to fetal hyperthyroidism. Obstet Gynecol 1996;87(5 Pt 2):838-840

8 Yanai N, Shveiky D. Fetal hydrops, associated with maternal propylthiouracil exposure, reversed by intrauterine therapy. Ultrasound Obstet Gynecol 2004;23(02):198-201

9 Bellini C, Hennekam RC. Non-immune hydrops fetalis: a short review of etiology and pathophysiology. Am J Med Genet A 2012; 158A(03):597-605

10 Ogilvy-Stuart AL. Neonatal thyroid disorders. Arch Dis Child Fetal Neonatal Ed 2002;87(03):F165-F171

11 Van Vliet G, Polak M, Ritzén EM. Treating fetal thyroid and adrenal disorders through the mother. Nat Clin Pract Endocrinol Metab 2008;4(12):675-682

12 Stagnaro-Green A, Abalovich M, Alexander E, et al; American Thyroid Association Taskforce on Thyroid Disease During Pregnancy and Postpartum. Guidelines of the American Thyroid Association for the diagnosis and management of thyroid disease during pregnancy and postpartum. Thyroid 2011;21(10):1081-1125 